\title{
Combining Textual and Speech Features in the NLI Task Using State-of-the-Art Machine Learning Techniques
}

\author{
Pavel Ircing, Jan Švec, Zbyněk Zajíc \\ University of West Bohemia \\ Univerzitní 8 \\ 30614 Plzeň \\ Czech Republic \\ \{ircing, honzas, zzajic\}@kky.zcu.cz
}

\author{
Barbora Hladká, Martin Holub \\ Charles University \\ Malostranské nám. 25 \\ 11800 Prague 1 \\ Czech Republic \\ \{hladka, holub\}@ufal.mff.cuni.cz
}

\begin{abstract}
We summarize the involvement of our CEMI team in the "NLI Shared Task 2017", which deals with both textual and speech input data. We submitted the results achieved by using three different system architectures; each of them combines multiple supervised learning models trained on various feature sets. As expected, better results are achieved with the systems that use both the textual data and the spoken responses. Combining the input data of two different modalities led to a rather dramatic improvement in classification performance. Our best performing method is based on a set of feed-forward neural networks whose hidden-layer outputs are combined together using a softmax layer. We achieved a macro-averaged F1 score of 0.9257 on the evaluation (unseen) test set and our team placed first in the main task together with other three teams.
\end{abstract}

\section{Native Language Identification}

We think of learning a second language $\mathrm{L} 2$ by people with their native language L1. The Native Language Identification (NLI) task is to recognize the L1 of an L2 author's text or speech. Most work in the NLI field has focused on identifying the native language of students learning English as a second language, which is also reflected in the very first experiments with written responses and spoken responses, see (Koppel et al., 2005) and (Schuller et al., 2016), respectively.

With respect to the form of analyzed responses, written ones and spoken ones, we distinguish between text-based NLI and speech-based NLI, respectively. In text-based NLI, all experiments per- formed so far are based on searching patterns in texts that are common to groups of speakers of the same L1. This idea naturally arises from general awareness that L1 speakers use typical grammatical constructions or make typical mistakes when using L2.

Speech-based NLI is naturally being approached differently, mainly by analyzing the acoustic properties of a speech utterance by the acoustic signal processing methods. Very recently (Schuller et al., 2016) organized the Native Language Sub-Challenge with spoken responses.

While most NLI research has focused on English as L2, there is also a growing trend to apply the techniques to other L2 languages, e.g. Norwegian (Malmasi et al., 2015a), Chinese (Malmasi and Dras, 2014a), Finnish (Malmasi and Dras, 2014b).

NLI has a wide variety of potential applications and both its techniques and findings can be used in areas such as Second-Language Acquisition (Ortega, 2009), author profiling (Rangel et al., 2013), and authorship contribution (Halvani et al., 2016). Typically, NLI is employed as a starting point for investigations into crosslinguistic influence, see e.g. (Jarvis and Paquot, 2012).

In this paper, we summarize the involvement of the CEMI team in the NLI Shared Task 2017 co-located with the 12th Workshop on Innovative Use of NLP for Building Educational Applications held in September 2017 in Copenhagen, Denmark. The NLI task is typically framed as a classification problem where the set of L1s is known a priori. The NLI Shared Task 2017 deals with 11 output classes $C=\{$ ARA, CHI, FRE, GER, HIN, ITA, JPN, KOR, SPA, TEL, TUR $\},{ }^{1}$ and defines three sub-tasks that differ in data sources available:

\footnotetext{
${ }^{1}$ The classes correspond to 11 different L1 languages, namely Arabic, Chinese, French, German, Hindi, Italian, Japanese, Korean, Spanish, Telugu, and Turkish, respectively.
} 


\begin{tabular}{lccc}
\hline & ICLEv2 & Lang-8 & TOEFL11 \\
& Granger et al. (2009) & Mizumoto et al. (2011) & Blanchard et al. (2013) \\
\hline number of documents & 6,085 & 154,702 & 12,100 \\
average document length & 617 & 150 & 348 \\
number of L1s & 16 & 65 & 11 \\
number of topics & variation & variation & 8 \\
proficiency level & inter, high & variation & low, inter, high \\
\hline
\end{tabular}

Table 1: Some of the NLI English textual datasets.

- ESSAY Task - the L1 identification is based solely on the written essays

- SPEECH Task - the L1 identification is based on the speech utterances (their transcripts and/or extracted i-vectors capturing the acoustic properties of the recorded speech)

- (Main) FUSION Task - the NLI system is allowed to use both sources listed above

We participated in each track and used only the available labelled data. The data collection consists of 13,200 English essays (written texts) and spoken responses (written transcriptions and preprocessed i-vectors) and its pairwise disjoint subsets of 11,000 training examples, 1,100 development test examples, and 1,100 evaluation test examples. Both training and development test sets were provided to the shared task participants, while the evaluation test set was the unseen data portion kept only for the final evaluation performed by the organizers. The i-vectors are computed from 45-second audio files corresponding to orthographic transcriptions. The results of the NLI Shared Task 2017 are reported in Malmasi et al. (2017).

In the rest of this paper, we first review related works in Section 2. Other works on feature engineering inspired us to choose features for our experiments. More details about the features we used are provided in Section 3. Our approach focuses mainly on different machine learning algorithms explained in Section 4. We design a two-step procedure consisting of training standalone classifiers (see Section 4.1), and training additional parameters of fused models (see Section 4.2). In total, we submitted three different system architectures described in Section 4.3. In Section 5 we present and discuss our results, and in the last Section 6 we make some final comments.

\section{Related work}

Text-based NLI has been addressed since 2005 and speech-based NLI since 2016. We give a picture of which results have been produced since the very beginning to date. Given the scope of the NLI Shared Task 2017, we focus on studies having English as a second language.

\subsection{Text-based NLI}

An exhaustive overview of NLI until 2014 has been provided by Massung and Zhai (2016). In Table 1 we show the basic characteristics of the datasets widely used so far. Now we mention only some works with respect to three milestones.

The very beginning Koppel et al. (2005) implemented a fully automated method to address text-based NLI for the first time ever. They experimented with the sub-part of the ICLEv2 corpus containing only five L1s. ${ }^{2}$ Their feature set included relative frequencies of function words, character $n$-grams, error types and rare POS bigrams so that each document was represented as a vector of 1,035 features. Their SVM-based method achieved just above $80 \%$ accuracy.

Seven years later There were three papers alone on text-based NLI at the COLING 2012 conference: Brooke and Hirst (2012) developed a robust model that works with $79.3 \%$ accuracy when used across the ICLEv 2 and Lang- 8 corpora. They extracted a set of 800,000 features, ${ }^{3}$ which was extremely large in comparison to the set used by Koppel et al. (2005). They also discuss the inadequacy of ICLEv2 as a training corpus and recommended to pay more attention to the overall validity of NLI experiments, rather than to

\footnotetext{
${ }^{2}$ Bulgarian, Czech, French, Russian, Spanish

${ }^{3}$ Function words, character $\{1-3\}$-grams, word $\{1-2\}$ grams, POS $\{1-3\}$-grams, context-free grammar production rules, dependencies, proper nouns.
} 
specific technical approaches. Bykh and Meurers (2012) experimented with ICLEv2 as well but their seven target classes were different from those used in (Brooke and Hirst, 2012). They explored recurring word and POS $n$-grams and they achieved $89.71 \%$ accuracy that was later surpassed by Tetreault et al. (2012) who used (Koppel et al., 2005)'s feature set enriched with the Tree Substitution Grammar features (Swanson and Charniak, 2012), the Stanford dependency features (de Marneffe et al., 2006) and language model perplexity scores to achieve an accuracy of $90.1 \%$.

The TOEFL11 corpus available The First Native Language Identification Shared Task in 2013 (Tetreault et al., 2013) marks an important stage in the text-based NLI research mainly because of making available the TOEFL11 corpus. This corpus consists of essays on eight different topics written by non-native speakers of three proficiency levels (low/medium/high); the essays' authors have 11 different native languages listed in Section 1. The corpus contains 1,100 essays per language with an average of 348 word tokens per essay. A corpus description and the motivation to build such corpus can be found in (Blanchard et al., 2013). The report by Tetreault et al. (2013) summarizes the techniques used and the results achieved by the competing teams in the shared task.

TOEFL11 has become a common evaluation resource for the text-based NLI task. Nicolai et al. (2013) used a subset of the corpus with only five L1s to train probabilistic graphical models. ${ }^{4}$ Bykh and Meurers (2014) systematically explored nonlexicalized and lexicalized context-free grammar production rules. They combined them with wordbased and POS-based $n$-grams and they achieved accuracy of $84.8 \%$, the best result reported by that time. Later on, Ionescu et al. (2014) obtained a new state-of-the-art result, $85.3 \%$ accuracy, so that they combined several string kernels using multiple kernel learning to do feature selection. Their method is completely language independent, and texts are treated as a sequence of characters.

Kríž et al. (2015) measure similarity between general English and English used by L1 speakers using cross-entropy scores, which then serve as features for an SVM classifier. It requires 12 language models of English - one model of general

\footnotetext{
${ }^{4}$ Chinese, French, German, Japanese, and Turkish.
}

\begin{tabular}{lcl}
\hline System & \# features & Acc. \\
\hline 1 (Malmasi and Dras, 2017) & $?$ & 85.3 \\
2 (Bykh and Meurers, 2016) & $?$ & $85.4^{\star}$ \\
3 (Gebre et al., 2013) & 73,626 & 84.6 \\
4 (Jarvis et al., 2013) & $400 \mathrm{~K}$ & 84.5 \\
5 (Ionescu et al., 2014) & $?$ & 84.1 \\
$\ldots$ & $\ldots$ & $\ldots$ \\
(Kríž et al., 2015) & 55 & 82.4 \\
\hline
\end{tabular}

Table 2: Top 5 written NLI systems on TOEFL11, and for comparison the system with the lowest number of (entropy-based) features. A 10-fold cross-validation accuracy is provided (Acc. in \%). ${ }^{\star}$ The authors report the $85.4 \%$ accuracy on the evaluation test set.

English based on Wikipedia data and eleven special models, each based on a particular L1 group. The best classification accuracy of $82.4 \%$ has been achieved by a combination of language models built upon four different n-gram types -- tokens, characters, suffixes, and POS tags. These 44 (= $4 \times 11)$ cross-entropy scores completed with other nine numerical and two categorical features result in the final set of 55 features. In fact, this compact feature set comprises a big amount of statistical information about a huge number of $n$-grams hidden in the language models consisting of smoothed linear $n$-grams combinations.

In contrast, (Malmasi and Cahill, 2015) extracted a much bigger feature set and they focused on measuring association between two feature sets through classification errors.

The very last work on text-based NLI focuses on systematic examination of ensemble methods for addressing NLI with three L2s, namely English, Norwegian, and Jinan Chinese (Malmasi and Dras, 2017).

Table 2 presents the top 5 text-based NLI systems on TOEFL11. We also provide the same figures for the system (Kríž et al., 2015) with an extremely low number of features. Here is a brief description of the algorithms and the features used:

- (Malmasi and Dras, 2017) - ensemble classifier, bagging, linear discriminant analysis; $n$-grams of lemmas, words, function words, POS tags, dependencies, CFG rules, Adaptor Grammar, TSG fragments

- (Bykh and Meurers, 2016) - ensemble classifier; $n$-grams of lemmas, words, POS tags 
where $1 \leq n \leq 10$, dependencies, suffixes, verb subcategorization patterns

- (Gebre et al., 2013) - SVM; tf-idf of word unigrams and bigrams, df $\geq 5$, normalized feature vectors

- (Jarvis et al., 2013) - SVM; $\{1,2,3\}$-grams of words, lemmas, POS tags, df $\geq 2$, normalized feature vectors

- (Ionescu et al., 2014) - Kernel-based learning; character $\{5-8\}$-grams

- (Kríž et al., 2015) - SVM; entropy-based features using language modeling (tokens, characters, POS, suffixes)

Malmasi et al. (2016) analyze the results of the Discriminating between Similar Languages shared task and they state that numerous teams attempted to use new deep learning-based approaches, and that most of them ended with a poor performance compared to traditional classifiers. To the best of our knowledge, there has been no published paper on using deep learning in textbased NLI yet. We can only speculate that researchers have already applied deep learning techniques to text-based NLI but they did not beat traditional classifiers.

\subsection{Speech-based NLI}

The speech-based NLI shared task was organized under the name Native Language Subchallenge as one of the subtasks of the INTERSPEECH 2016 Computational Paralinguistics Challenge (Schuller et al., 2016).

The ETS Corpus of Non-native Spoken English was provided for the task consisting of 5,132 examples in total $-3,300$ examples were selected for training, 965 examples for the development test set, and 867 examples for the evaluation test set. The corpus includes spoken responses from non-native speakers of English drawn from 11 different L1 backgrounds that are identical to the TOEFL11 L1s. The recorded utterances are 45second long for each speaker. The participants were provided with the audio files (amplitude normalized) and were also pointed to the toolkit that was used to extract the audio features for the baseline system provided by the sub-challenge organizers. It is obvious that the extracted features did not reflect only the actual content of the utterances but also - and possibly more prominently - the

\begin{tabular}{ll}
\hline System & UAR $(\%)$ \\
\hline 1 (Abad et al., 2016) & 84.6 \\
2 (Shivakumar et al., 2016) & 78.6 \\
3 (Gosztolya et al., 2016) & 70.7 \\
4 (Huckvale, 2016) & 69.8 \\
5 (Senoussaoui et al., 2016) & 68.4 \\
6 (Keren et al., 2016) & 61.5 \\
7 (Jiao et al., 2016) & 52.2 \\
8 (Rajpal et al., 2016) & 39.8 \\
\hline baseline & 45.1 \\
\hline
\end{tabular}

Table 3: Spoken NLI systems submitted to the 2016 NLI shared task. UAR stands for Unweighted Average Recall.

acoustic properties of the speech that are supposedly and significantly influenced by the speaker's native language. Given the usual background of the INTERSPEECH attendees, it is only natural that most participants of the sub-challenge had a strong background in speech signal processing and (at least the top teams) concentrated on their own sophisticated methods for feature extraction.

According to our knowledge, no transcriptions of the recorded utterances were provided and none of the participants attempted to use an automatic speech recognition system in order to create transcripts that could be used as the source of textual features. Given the poor performance of the system based solely on the (manual) speech transcriptions in the NLI Shared Task 2017, it seems that ignoring the textual content of the utterances was a wise decision.

Table 3 presents the systems submitted to the sub-challenge. Since the top two teams, whose systems outperformed the rest by a large margin, employed the i-vector feature representation, the organizers have decided to provide the i-vectors directly to the NLI Shared Task 2017 participants, supposedly in order to lower the entry threshold for participants without the speech processing background. A short high-level description of the $\mathrm{i}$-vector principles is given in Section 3.

\section{Feature extraction}

Textual features Since our work concentrates mainly on the different machine learning algorithms (described in detail in the later sections), we did not perform any sophisticated feature engineering. Instead, we picked the textual 
features that have been proven to be effective in the experiments performed by other researchers previously, being mostly inspired by Gebre et al. (2013). We have employed $n$-grams of various lengths from the following "data streams":

- Word unigrams, bigrams and trigrams extracted from both essays and speech transcriptions.

- Character n-grams with $n$ ranging from 3 to 5 , extracted from the essays only.

- POS n-grams with $n$ ranging from 1 to 5, also extracted only from the essays.

All features were weighted using the wellknown $t$-idf weighting scheme, with the sublinear If scaling and the standard $i d f$, that is, the weight $w$ of each feature $i$ in the document $j$ is given by:

$$
w_{i, j}=\left(1+\log \left(t f_{i, j}\right)\right) \cdot \log \frac{N}{n_{i}}
$$

where $N$ denotes the total number of documents and $n_{i}$ the number of documents containing the feature $i$. Then the resulting feature vectors are normalized to unit length. Quick experiments on the development data have shown that:

- Sublinear if scaling substantially outperforms the unscaled $t f$.

- The number of n-gram based-features used in the classification can be reduced to top 30,000 features (ordered by decreasing $t f$ ) without hurting the performance. ${ }^{5}$ The feature vector dimension was thus limited to $30 \mathrm{k}$ for all textual features described above.

Speech features Here we did not have any other choice than using the i-vectors provided by the Shared Task organizers. The i-vectors were originally developed as a representation of speech utterances in a low-dimensional subspace, which efficiently conveys speaker's "vocal" characteristics and is therefore suitable for speaker recognition (Dehak et al., 2011). The i-vectors of course contain also the information about the acoustic environment, transmission channel or phonetic content of the utterance. Intuitively, the phonetic content appears to be an important factor distinguishing

\footnotetext{
${ }^{5}$ Note that the total number of features would exceed 2.5 million in the case of word trigrams.
}

the L1 of the speaker as the native language naturally influences the way the speaker pronounces English phonemes. The i-vectors were extracted from the 45 -second audio files by the task organizers, employing a state-of-the-art approach and using the Kaldi ${ }^{6}$ toolkit. The dimension of the ivectors is 800 , reduced by factor analysis from supervector of statistics accumulated on the universal background model with 1,024 components.

Several experiments (and the description of the the state-of-the-art NLI in (Malmasi and Dras, 2017)) confirmed our intuition that simply concatenating the individual feature vectors and training a single classifier does not yield the best results. We therefore concentrated mainly on the development of the fused (ensemble) classifiers, described in details in the following section.

Finally, let us point out that we have decided not to use the character and POS n-grams from the speech transcription data in our final systems. The reason is the fact that 1 ) word n-grams are by far the best performing textual features, yet their performance was rather poor on the speech transcriptions, and 2) any performance gain from character and POS n-grams was clearly overshadowed by the i-vectors contribution in both speech and fusion tasks.

\section{Prediction model}

We used multiple supervised models to process each type of input features. Then, we fused the predictions of such models, i.e. we combined the outputs of the classifiers instead of combining the input features and training one joint model. This approach consists of two steps: (1) training the stand-alone classifiers, and (2) training the additional parameters of the fused model. Optionally, the step (2) could employ additional retraining of the stand-alone classifiers.

\subsection{Stand-alone classifiers}

The term "stand-alone classifiers" is herein used for the systems whose internal parameters are trained with a standard supervised machine learning algorithm (e.g., gradient descent) and which take the input feature vector and output a vector of $|C|$ probabilities. The decision about the class membership is then determined solely by the index of the maximum value of such output vector.

\footnotetext{
${ }^{6}$ http://kaldi-asr.org
} 
Linear models To perform the classification using textual features, we widely used linear models. The training procedure of such model varied - we experimented with a linear SVM and stochastic gradient descent training implemented using the LinearSVC and SGDClassifier classes from the scikit-learn toolkit (Pedregosa et al., 2011). Both implementations support sparse feature representation and therefore in our experiments the full feature vector could be used.

Non-linear models We also used non-linear models implemented as feed-forward neural networks (FFNN) containing hidden layers with nonlinear functions. In our experiments we also tried the very deep architectures such as ResNets and DenseNets, but they were outperformed by a relatively simple FFNN with one hidden layer. This is probably caused by a relatively low number of training examples and a high number of parameters of deeper networks. The FFNNs were used to classify both textual and speech-related features. The size of the textual feature vectors was reduced to $30 \mathrm{k}$ as explained in Section 3. The FFNNs were implemented in the Keras system (Chollet et al., 2015). To optimize the FFNNs, we used the ADAM algorithm (Kingma, 2015) with a categorical cross-entropy loss.

Probabilistic Linear Discriminant Analysis (PLDA) is a state-of-the-art system for i-vector based speaker verification (Kenny, 2005) and can by easily used for representation of another information, the L1 in our case. I-vectors also contain some noisy information not relevant to the L1 identity (e.g. influence of the channel, speaker etc.). If structured training data (more than one session for each L1) are available, PLDA can be trained to model L1 and session variability separately. Then, only the L1 domain is used for identification. Moreover, the PLDA model itself can be used as a powerful tool for compute the similarity between two i-vectors (only in L1 domain). In our case, the test i-vector is compared to $|C|$ L1 i-vectors representing the models of particular L1 languages. The similarities are normalized to sum up to one. The $\mathrm{L} 1 \mathrm{i}$-vector is computed as the mean of all i-vectors belonging to a given class. The PLDA classifier was used to classify i-vector features in the ensemble systems used in the SPEECH and FUSION tasks.

\subsection{Model combinations}

To combine the outputs of the stand-alone classifiers, we experimented with three different schemas: (1) discriminative logistic regression, (2) softmax combination of hidden layer's outputs, and (3) softmax combination of classifier's outputs. Since the development data set provides an additional valuable source of labelled data, special attention has to be paid to the correct estimation of the fusion parameters, as described below.

Discriminative logistic regression for fusing system's outputs was implemented using an open-source FoCal Multi-class toolkit (Brümmer, 2007). This MATLAB toolkit allows evaluation, calibration and fusion of, and decision-making with, multi-class statistical pattern recognition scores. This toolkit is different from, but similar in design principles to the original FoCal Toolkit that was used by several NIST Speaker Recognition Evaluation 2006 participants to fuse and calibrate their scores (Brümmer et al., 2007). For the fusion we used the tool based on calibration and discriminative logistic regression of $K$ classifiers

$$
\hat{\boldsymbol{y}}(x)=\sum_{k=1}^{K} \alpha_{k} \boldsymbol{y}_{k}(x)+\boldsymbol{\beta},
$$

where $\boldsymbol{y}_{k}(x) \in \Re^{|C|}$ is a vector of posterior probabilities obtained from $k$-classifier, $\hat{\boldsymbol{y}}(x)$ is a vector of fused probabilities and vectors $\boldsymbol{\alpha} \in \Re^{K}$ and $\boldsymbol{\beta} \in \Re^{|C|}$ are parameters of the fusion. These parameters were first estimated on the held-out data (data not used to train the stand-alone classifiers), then the classifiers were retrained to employ all available labelled data (train and development) and the previously estimated vectors $\boldsymbol{\alpha}$ and $\boldsymbol{\beta}$ were used.

Softmax combination The softmax combination is implemented as a neural network without hidden layers. The vector of fused probabilities $\hat{\boldsymbol{y}}(x)$ is given by:

$$
\begin{gathered}
\boldsymbol{a}(x)=\boldsymbol{W} \cdot\left(\begin{array}{c}
\boldsymbol{y}_{1}(x) \\
\vdots \\
\boldsymbol{y}_{K}(x)
\end{array}\right)+\boldsymbol{b} \\
\hat{\boldsymbol{y}}(x)=\operatorname{softmax}(\boldsymbol{a}(x))
\end{gathered}
$$

where $\boldsymbol{W}$ is a weight matrix and $\boldsymbol{b}$ is a bias vector. The values of $\boldsymbol{W}$ and $\boldsymbol{b}$ are optimized using 


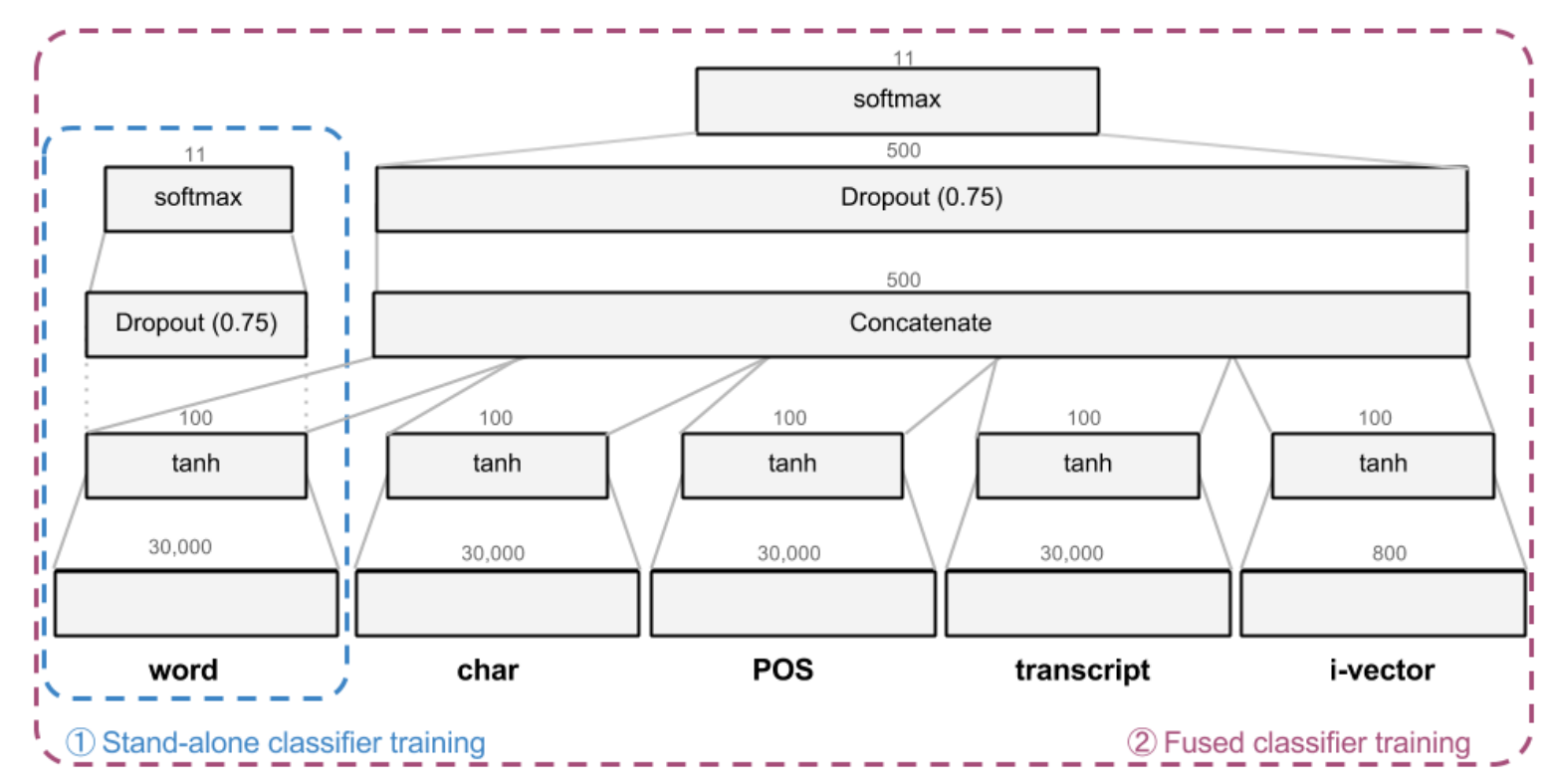

Figure 1: Architecture of the homogeneous neural network for the FUSION task.

the ADAM algorithm and the categorical crossentropy loss. We experimented with two different choices of $\boldsymbol{y}_{k}$ :

- The output of the hidden layer from the FFNN corresponding to a specific feature set. In this case, we merged the trained standalone FFNNs to form a fused FFNN according to Figure 1 and the parameters of the stand-alone FFNNs were trained using the back-propagation errors. The stand-alone FFNNs and the fused FFNN were trained on the union of train and development datasets.

- The $|C|$-dimensional output of the standalone classifier. For the linear models the output consists of the values of decision functions, for the FFNN such output is the potential of the output layer before applying the softmax activation. In this case, we first trained the stand-alone classifiers on the train dataset, and then we trained just the fusion parameters $W$ and $b$ on the development dataset.

\subsection{Submitted systems}

Based on the experiments with the development data set, we finally decided to submit three different system architectures. Each architecture is a combination of multiple systems trained on different features, even in the ESSAY and SPEECH tasks.

- Classical model ensemble ("ensemble") consists of different stand-alone models trained separately and combined using the discriminative logistic regression.

- Homogeneous FFNN (“homogeneous") uses a set of stand-alone FFNNs trained separately. The number of hidden layers, number of neurons in hidden layers, and activation functions are identical for each standalone FFNN. The outputs of hidden layers in the trained FFNNs are combined using softmax combination. The resulting network is retrained. To avoid overfitting, we used the dropout layer before the softmax layer.

- Heterogeneous FFNN ("heterogeneous") employs a set of FFNNs with different architectures. The stand-alone classifiers are trained separately using different objectives. The $|C|$-dimensional outputs are then combined using softmax combination. The resulting network is not retrained during estimating the softmax weights and biases.

For different tasks we used the following different sets of features and classifiers:

ESSAY task - the ensemble system used word, char and POS features and FFNN and SGDClassifier models for each feature set (= $3 \times 2$ stand-alone models). The homogeneous system used word, char and POS features and FFNN with 1 hidden layer containing 100 neurons. The heterogeneous system used the same features and SGDClassifier only. 


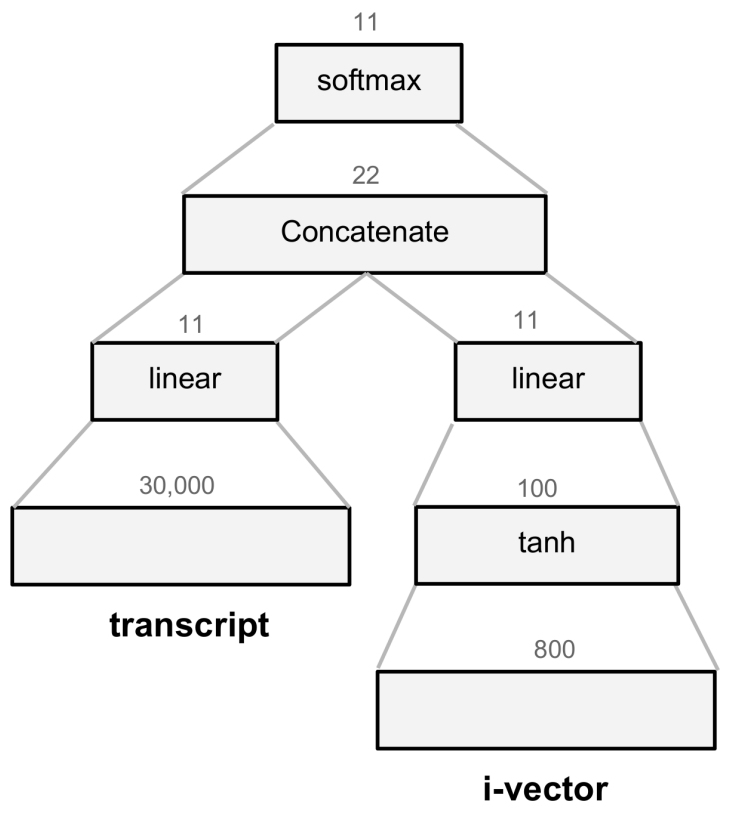

Figure 2: Architecture of the heterogeneous neural network for the SPEECH task.

SPEECH task - the ensemble system used FFNN classifiers trained on word and char features extracted from transcripts and PLDA and FFNN trained from i-vectors. The homogeneous system used word features from transcripts and i-vectors and FFNN (1 hidden layer, 100 neurons). The heterogeneous system contained SGDClassifier trained from transcript word features and FFNN (1 hidden layer, 100 neurons) trained on i-vectors (see Figure 2).

FUSION task - for each system we used a combination of the stand-alone classifiers used in the ESSAY and SPEECH tasks. An example of such a combination for the homogeneous system is given in Figure 1.

\section{Results and discussion}

The final results of the submitted systems measured on the unseen evaluation test set are shown in Table 4. In this paper, all F1 values are macroaveraged over all 11 output classes. It should be noted that the relatively low number of test examples combined with a higher number of classes resulted in quite wide confidence intervals. For example, we evaluated the F1 measure for the homogeneous system on the FUSION task. Using the development data set and a bootstrapping approach with 550 samples and 1,000 repetitions we found that the resulting average F1 0.9112 has associated a $95 \%$ confidence interval of $<0.8850$;

\begin{tabular}{lll}
\hline Task & System & F1 \\
\hline \multirow{3}{*}{ ESSAY } & ensemble & $\mathbf{0 . 8 5 3 6}$ \\
& homogeneous & 0.8491 \\
& heterogeneous & 0.8464 \\
\hline \multirow{3}{*}{ SPEECH } & ensemble & 0.8570 \\
& homogeneous & 0.7987 \\
& heterogeneous & $\mathbf{0 . 8 6 0 7}$ \\
\hline \multirow{3}{*}{ FUSION } & ensemble & 0.9238 \\
& homogeneous & $\mathbf{0 . 9 2 5 7}$ \\
& heterogeneous & 0.9244 \\
\hline
\end{tabular}

Table 4: Summary of the results for each task and our three architectures. The macro-averaged F1 value was measured on the unseen evaluation test set by the shared task organizers.

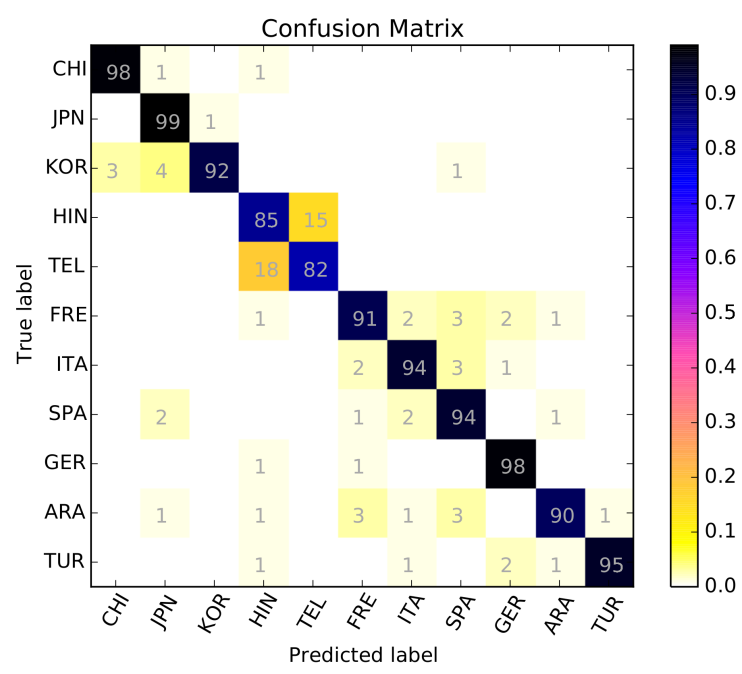

Figure 3: Confusion matrix for the FUSION task, homogeneous system. Measured on the unseen evaluation test set.

$0.9345>(!)$. Even the variations caused by the random seed selection are noticeable: for ten different seeds the $\mathrm{F} 1$ value varies between 0.9075 and 0.9166 . For proper perspective, it is important to keep in mind that the difference of 0.001 in F1 evaluated on test data means that the systems mismatch in approximately 1 correctly classified test example.

Table 4 also shows another interesting fact that the F1 value in the SPEECH task is higher than in the ESSAY task. We assume this is caused by the availability of two modalities - the speech alone (i-vectors) and the lexical information (transcripts). On the development test set, the stand-alone classifier trained solely on i-vectors 
achieved an F1 value of only 0.8080 , while the classifier trained solely on transcribed text features achieved only 0.5787. In this case, the combination of a relatively weak predictor with a strong model further improved the performance to 0.8610. We also observed that training classifiers on the union of the training and development data sets consistently improves performance - the increase in the F1 value (evaluated on the unseen test data) is approximately 0.004 . To illustrate the performance on different feature types, we evaluated the stand-alone classifiers of the homogeneous system trained for the FUSION task on the development data. The results are summarized in Table 5.

We also used the Local Interpretable Modelagnostic Explanations (LIME) method (Ribeiro et al., 2016) to extract the most informative features for a given L1 class. The results showed that just the presence of certain words very often leaks significant information about the L1 language (this effect was already observed by (Gebre et al., 2013)) - for example essays labelled as JPN contain words Japan, Japanese, KOR mention Korea and Korean. Also, there are some typos that have origin in the L1 language (e.g., ITA: pubblic from Italian pubblico - 52 examples in the training data, FRE: exemple from French exemple - 174 examples). The confusion matrix in Figure 3 shows that $40 \%$ of all errors are confusions between the HIN and TEL classes. This is probably caused by the fact that the L1 speakers of these languages have gone through the same educational system of India. In addition, the geographic references mentioned above do not allow to discriminate between them. During the system development, we also experimented with the advanced architectures of neural networks, such as convolutional networks, recurrent networks, ResNets, DenseNets and pretrained word embeddings but none of them performed better than the linear SVM baseline.

\section{Conclusion}

Malmasi et al. (2015b) previously showed that even NLI systems working with just written essays can outperform human decisions. Our experiments revealed that adding information extracted from the spoken responses of non-native English speakers results into a substantial improvement in

\begin{tabular}{lll}
\hline & Features & F1 \\
\hline \multirow{3}{*}{ ESSAY } & word & 0.8151 \\
& char & 0.8025 \\
& POS & 0.5012 \\
\hline \multirow{2}{*}{ SPEECH } & transcript words & 0.5591 \\
& i-vectors & 0.7962 \\
\hline
\end{tabular}

Table 5: Performance of five stand-alone classifiers used in the homogeneous FUSION system measured on the development test set. The standalone classifiers are FFNNs, 1 hidden layer with 100 neurons. In the FUSION model they were further trained by the softmax combination training.

classification performance (about $5 \%$ relative $^{7}$ ). It corroborates our initial intuition that the textual and spoken data really complement well as the source of information about the L1 language.

To sum up our results measured on the unseen evaluation test set, we attained the following macro-averaged F1 scores:

- ESSAY task: 0.8536

- shared second place in the task,

- SPEECH task: 0.8607

- shared first place in the task,

- main FUSION task: 0.9257

- shared first place in the task.

Let us stress out that those results were achieved by rather straightforward (yet at the same time informed and careful) application of state-of-the-art machine learning algorithms, using feature extraction methods that have already been proven efficient both in previous NLI shared tasks and in our NLP and speech processing research.

\section{Acknowledgements}

We really appreciate the hard work done by the organizers. They prepared the high-quality data that motivated the participants to work on an interesting project. This research was supported by the Grant Agency of the Czech Republic, projects No. GAČR GBP103/12/G084 and ID 16-10185S, and by the Charles University project No. SVV 260333.

\footnotetext{
${ }^{7}$ Measured as the relative difference between our best system using both text and speech data and the best system based solely on essays submitted to the NLI Shared Task 2017.
} 


\section{References}

Alberto Abad, Eugénio Ribeiro, Fábio Kepler, Ramon Astudillo, and Isabel Trancoso. 2016. Exploiting Phone Log-Likelihood Ratio Features for the Detection of the Native Language of Non-Native English Speakers. In Interspeech 2016. pages 2413-2417.

Daniel Blanchard, Joel Tetreault, Derrick Higgins, Aoife Cahill, and Martin Chodorow. 2013. TOEFL11: A Corpus of Non-Native English. ETS Research Report Series 2013(2):i-15.

Julian Brooke and Graeme Hirst. 2012. Robust, Lexicalized Native Language Identification. In Proceedings of COLING 2012. Mumbai, India, pages 391408.

Niko Brümmer. 2007. Focal Multi-Class: Toolkit for Evaluation, Fusion and Calibration of Multi-Class Recognition Scores. In Spescom DataVoice.

Niko Brümmer, Lukáš Burget, Jan Černocký, Ondřej Glembek, František Grézl, Martin Karafiát, David Leeuwen van, Pavel Matějka, Petr Schwarz, and Albert Strasheim. 2007. Fusion of heterogeneous speaker recognition systems in the stbu submission for the nist speaker recognition evaluation 2006. IEEE Transactions on Audio, Speech, and Language Processing 15(7):2072-2084.

Serhiy Bykh and Detmar Meurers. 2012. Native Language Identification using Recurring $n$-grams - Investigating Abstraction and Domain Dependence. In Proceedings of COLING 2012. Mumbai, India, pages $425-440$.

Serhiy Bykh and Detmar Meurers. 2014. Exploring Syntactic Features for Native Language Identification: A Variationist Perspective on Feature Encoding and Ensemble Optimization. In Proceedings of COLING 2014: Technical Papers. Dublin City University and ACL, Dublin, Ireland, pages 1962-1973.

Serhiy Bykh and Detmar Meurers. 2016. Advancing Linguistic Features and Insights by Label-informed Feature Grouping: An Exploration in the Context of Native Language Identification. In Proceedings of COLING 2016, the 26th International Conference on Computational Linguistics: Technical Papers. The COLING 2016 Organizing Committee, Osaka, Japan, pages 739-749.

François Chollet et al. 2015. Keras. https:// github.com/fchollet/keras.

Marie-Catherine de Marneffe, Bill MacCartney, and Christopher D. Manning. 2006. Generating Typed Dependency Parses from Phrase Structure Parses. In Proceedings of the Fifth International Conference on Language Resources and Evaluation. pages 449454.
Najim Dehak, Patrick J Kenny, Réda Dehak, Pierre Dumouchel, and Pierre Ouellet. 2011. Front-End Factor Analysis for Speaker Verification. IEEE Transactions on Audio, Speech, and Language Processing 19(4):788-798.

Binyam Gebrekidan Gebre, Marcos Zampieri, Peter Wittenburg, and Tom Heskes. 2013. Improving Native Language Identification with TF-IDF Weighting. In Proceedings of the Eighth Workshop on Innovative Use of NLP for Building Educational Applications. ACL, Atlanta, Georgia, pages 216-223.

Gábor Gosztolya, Tamás Grósz, Róbert Busa-Fekete, and László Tóth. 2016. Determining Native Language and Deception Using Phonetic Features and Classifier Combination. In Interspeech 2016. pages 2418-2422.

Sylviane Granger, Estelle Dagneaux, Fanny Meunier, and Magali Paquot. 2009. International Corpus of Learner English v2 (Handbook $+C D$ $R O M$ ). Presses universitaires de Louvain, Louvainla-Neuve.

Oren Halvani, Christian Winter, and Anika Pflug. 2016. Authorship Verification for Different Languages, Genres and Topics. Digital Investigation 16, Supplement:S33 - S43. \{DFRWS 2016 EuropeProceedings of the Third Annual $\{$ DFRWS $\}$ Europe.

Mark Huckvale. 2016. Within-Speaker Features for Native Language Recognition in the Interspeech 2016 Computational Paralinguistics Challenge. In Interspeech 2016. pages 2403-2407.

Radu Tudor Ionescu, Marius Popescu, and Aoife Cahill. 2014. Can Characters Reveal Your Native Language? A Language-Independent Approach to Native Language Identification. In Proceedings of the 2014 Conference on EMNLP. ACL, Doha, Qatar, pages 1363-1373.

Scott Jarvis, Yves Bestgen, and Steve Pepper. 2013. Maximizing Classification Accuracy in Native Language Identification. In Proceedings of the Eighth Workshop on Innovative Use of NLP for Building Educational Applications. ACL, Atlanta, Georgia, pages 111-118.

Scott Jarvis and Magali Paquot. 2012. Exploring the Role of $n$-grams in L1 Identification. In Scott Jarvis and Scott A. Crossley, editors, Approaching Transfer through Text Classification: Explorations in the Detection-based Approach, Bristol, UK: Multilingual Matters, pages 71-105.

Yishan Jiao, Ming Tu, Visar Berisha, and Julie Liss. 2016. Accent Identification by Combining Deep Neural Networks and Recurrent Neural Networks Trained on Long and Short Term Features. In Interspeech 2016. pages 2388-2392.

Patrick Kenny. 2005. Joint Factor Analysis of Speaker and Session Variability: Theory and Algorithms. Technical report, Centre de Recherche Informatique de Montreal. 
Gil Keren, Jun Deng, Jouni Pohjalainen, and Björn Schuller. 2016. Convolutional Neural Networks with Data Augmentation for Classifying Speakers' Native Language. In Interspeech 2016. pages 2393 2397.

Diederik P Kingma. 2015. ADAM: A Method for Stochastic Optimization pages 1-15.

Moshe Koppel, Jonathan Schler, and Kfir Zigdon. 2005. Determining an Author's Native Language by Mining a Text for Errors. In Proceedings of the Eleventh ACM SIGKDD International Conference on Knowledge Discovery in Data Mining. ACM, New York, NY, USA, KDD ’05, pages 624-628.

Vincent Kríž, Martin Holub, and Pavel Pecina. 2015. Feature Extraction for Native Language Identification Using Language Modeling. In Galia Angelova, Kalina Boncheva, and Ruslan Mitkov, editors, Proceedings of Recent Advances in Natural Language Processing. Hisarja, Bulgaria, pages 298-306.

Shervin Malmasi and Aoife Cahill. 2015. Measuring Feature Diversity in Native Language Identification. In Proceedings of the Tenth Workshop on Innovative Use of NLP for Building Educational Applications. ACL, Denver, Colorado, pages 49-55.

Shervin Malmasi and Mark Dras. 2014a. Chinese Native Language Identification. In Proceedings of the EACL-14. ACL, Gothenburg, Sweden, pages 95-99.

Shervin Malmasi and Mark Dras. 2014b. Finnish Native Language Identification. In Proceedings of the Australasian Language Technology Workshop (ALTA). Melbourne, Australia, pages 139-144.

Shervin Malmasi and Mark Dras. 2017. Native Language Identification using Stacked Generalization. CoRR abs/1703.06541.

Shervin Malmasi, Mark Dras, and Irina Temnikova. 2015a. Norwegian Native Language Identification. In Proceedings of RANLP 2015. ACL, Hissar, Bulgaria, pages 404-412.

Shervin Malmasi, Keelan Evanini, Aoife Cahill, Joel Tetreault, Robert Pugh, Christopher Hamill, Diane Napolitano, and Yao Qian. 2017. A Report on the 2017 Native Language Identification Shared Task. In Proceedings of the 12th Workshop on Building Educational Applications Using NLP. Association for Computational Linguistics, Copenhagen, Denmark.

Shervin Malmasi, Joel Tetreault, and Mark Dras. 2015b. Oracle and Human Baselines for $\mathrm{Na}$ tive Language Identification. In Proceedings of the Tenth Workshop on Innovative Use of NLP for Building Educational Applications. Association for Computational Linguistics, Denver, Colorado, pages 172-178.
Shervin Malmasi, Marcos Zampieri, Nikola Ljubešić, Preslav Nakov, Ahmed Ali, and Jörg Tiedemann. 2016. Discriminating between Similar Languages and Arabic Dialect Identification: A Report on the Third DSL Shared Task. In Proceedings of the Third Workshop on NLP for Similar Languages, Varieties and Dialects (VarDial3). The COLING 2016 Organizing Committee, Osaka, Japan, pages 1-14.

Sean Massung and ChengXiang Zhai. 2016. NonNative Text Analysis: A Survey. Natural Language Engineering 22(2):163-186.

Tomoya Mizumoto, Mamoru Komachi, and Masaaki Nagata. 2011. Mining Revision Log of Language Learning SNS for Automated Japanese Error Correction of Second Language Learners. In In Proceedings of the Fifth International Joint Conference on Natural Language Processing. pages 147-155.

Garrett Nicolai, Md Asadul Islam, and Russ Greiner. 2013. Native Language Identification Using Probabilistic Graphical Models. In International Conference on Electrical Information and Communication Technology (EICT). Khulna, Bangladesh, pages 0-1.

Lourdes Ortega. 2009. Understanding Second Language Acquisition / Rod Ellis. Hodder Education, Oxford, UK.

F. Pedregosa, G. Varoquaux, A. Gramfort, V. Michel, B. Thirion, O. Grisel, M. Blondel, P. Prettenhofer, R. Weiss, V. Dubourg, J. Vanderplas, A. Passos, D. Cournapeau, M. Brucher, M. Perrot, and E. Duchesnay. 2011. Scikit-learn: Machine learning in Python. Journal of Machine Learning Research 12:2825-2830.

Avni Rajpal, Tanvina B. Patel, Hardik B. Sailor, Maulik C. Madhavi, Hemant A. Patil, and Hiroya Fujisaki. 2016. Native Language Identification Using Spectral and Source-Based Features. In Interspeech 2016. pages 2383-2387.

Francisco Rangel, Paolo Rosso, Moshe Moshe Koppel, Efstathios Stamatatos, and Giacomo Inches. 2013. Overview of the Author Profiling Task at PAN 2013. In CLEF Conference on Multilingual and Multimodal Information Access Evaluation. CELCT, pages 352-365.

Marco Tulio Ribeiro, Sameer Singh, and Carlos Guestrin. 2016. "Why Should I Trust You?": Explaining the Predictions of Any Classifier. In Proceedings of the 22Nd ACM SIGKDD International Conference on Knowledge Discovery and Data Mining. ACM, New York, NY, USA, KDD '16, pages 1135-1144.

Björn Schuller, Stefan Steidl, Anton Batliner, Julia Hirschberg, Judee K. Burgoon, Alice Baird, Aaron Elkins, Yue Zhang, Eduardo Coutinho, and Keelan Evanini. 2016. The INTERSPEECH 2016 Computational Paralinguistics Challenge: Deception, Sincerity and Native Language. In Interspeech 2016. pages 2001-2005. 
Mohammed Senoussaoui, Patrick Cardinal, Najim Dehak, and Alessandro L. Koerich. 2016. Native Language Detection Using the I-Vector Framework. In Interspeech 2016. pages 2398-2402.

Prashanth Gurunath Shivakumar, Sandeep Nallan Chakravarthula, and Panayiotis Georgiou. 2016. Multimodal Fusion of Multirate Acoustic, Prosodic, and Lexical Speaker Characteristics for Native Language Identification. In Interspeech 2016. pages 2408-2412.

Ben Swanson and Eugene Charniak. 2012. Native Language Detection with Tree Substitution Grammars. In Proceedings of the 50th Annual Meeting of the Association for Computational Linguistics: Short Papers - Volume 2. Association for Computational Linguistics, Stroudsburg, PA, USA, ACL '12, pages 193-197.

Joel Tetreault, Daniel Blanchard, and Aoife Cahill. 2013. A Report on the First Native Language Identification Shared Task. In Proceedings of the Eighth Workshop on Innovative Use of NLP for Building Educational Applications. ACL, Atlanta, Georgia, pages 48-57.

Joel Tetreault, Daniel Blanchard, Aoife Cahill, and Martin Chodorow. 2012. Native Tongues, Lost and Found: Resources and Empirical Evaluations in Native Language Identification. In Proceedings of COLING 2012. The COLING 2012 Organizing Committee, Mumbai, India, pages 2585-2602. 